\title{
A novel approach in creating nephrostomy using a double-lumen access sheath during endoscopic combined intrarenal surgery
}

\author{
Rei Unno, Kazumi Taguchi, Shuzo Hamamoto, Tatsuya Hattori, Kengo Kawase, Tomoki Okada, \\ Ryosuke Chaya, Yutaro Tanaka, Teruaki Sugino, Taiki Kato, Toshiki Etani, Ryosuke Ando, \\ Atsushi Okada, Takahiro Yasui
}

Department of Nephro-urology, Nagoya City University Graduate School of Medical Sciences, Aichi, Japan

Contributions: (I) Conception and design: R Unno, K Taguchi; (II) Administrative support: T Yasui; (III) Provision of study materials or patients: R Unno, K Taguchi, S Hamamoto, A Okada; (IV) Collection and assembly of data: R Unno, K Taguchi, S Hamamoto, T Hattori, K Kawase, T Okada, R Chaya, Y Tanaka, T Sugino, T Kato, T Etani; (V) Data analysis and interpretation: R Unno, K Taguchi, R Ando; (VI) Manuscript writing: All authors; (VII) Final approval of manuscript: All authors.

Correspondence to: Kazumi Taguchi. Department of Nephro-urology, Nagoya City University Graduate School of Medical Sciences, 1 Kawasumi, Mizuho-cho, Mizuho-ku, Nagoya 467-8601, Japan. Email: ktaguchi@med.nagoya-cu.ac.jp.

\begin{abstract}
Background: This study aimed to evaluate the safety and efficacy of an inner ureteral access sheath (i-UAS) with a double-lumen channel used in ureteroscopic lithotripsy (URS) as a dilator for the percutaneous tract in endoscopic combined intrarenal surgery (ECIRS).

Methods: This was a single-center cohort study conducted from January 2016 to April 2020. We used an i-UAS as a dilator and a double-lumen catheter to insert a safety guidewire during the creation of the nephrostomy tract in ECIRS. Univariate and multivariate analyses were performed to assess the association between the perioperative parameters and the use of i-UAS. The primary endpoint was perioperative complications, and secondary endpoints were stone-free rate (SFR), operative time, fluoroscopy time, and duration of hospitalization.

Results: In total, 221 patients were enrolled during the study period. Patients were divided into an i-UAS dilation group $(n=108)$ and a one-shot dilation group $(n=113)$. No differences were observed between the two groups in terms of patient history. Univariate analyses indicated that, in the i-UAS dilation group, the operative time was shorter [105.50 (83.75-143.25) vs. $121.00(90.00-155.00) \mathrm{min} ; \mathrm{P}=0.02]$ and the modified Valdivia position was more frequently selected. Multivariate analyses showed no significant differences in the frequency of complications, such as urinary injury or postoperative pyelonephritis, but it showed a significantly shorter operative time as well as fewer tract creation troubles in the i-UAS dilation group.

Conclusions: Using an i-UAS as a dilator and a double-lumen catheter to insert a safety guidewire during ECIRS is a convenient and safe technical method for creating a nephrostomy tract that can reduce the operative time.
\end{abstract}

Keywords: Nephrostomy; ureteroscopy; endoscopic combined intrarenal surgery (ECIRS); percutaneous nephrolithotomy (PCNL); lithotripsy

Submitted Jul 15, 2021. Accepted for publication Oct 29, 2021.

doi: 10.21037/tau-21-611

View this article at: https://dx.doi.org/10.21037/tau-21-611

\section{Introduction}

Endoscopic combined intrarenal surgery (ECIRS) combines flexible ureteroscopic lithotripsy (fURS) and percutaneous nephrolithotomy (PCNL). Although it yields a high stone- free rate (SFR) $(1,2)$ severe complications, such as urinary injury and bleeding, can often occur (3-6). Gaining renal access is the most critical step for effective and safe PCNL or ECIRS. However, complications often occur during the 
creation of the percutaneous tract. Advances in available equipment have produced minimally invasive percutaneous tracts, which reduce the risk of complications (7-10). We have previously reported surgical techniques for preventing complications in ECIRS using a thin needle (22 gauge), which under ultrasound (US) and ureteroscopic lithotripsy (URS) guidance, helped suppress bleeding (11). We have also reported that the use of real-time virtual sonography is effective and is associated with a lower incidence of bleeding-related complications (12).

Although these technical improvements have been beneficial in reducing complications, some risks of bleeding and urinary injury remain. Procedures that could cause complications when creating a tract include inserting the main tract after guidewire placement and placing a safety (second) guidewire. The current one-shot dilation method has been reported to be convenient and safe but requires experience (13-15). Furthermore, insertion of the safety guidewire requires removal and re-insertion of the tract, increasing the risk of complications, particularly when lessexperienced doctors perform the procedure. Although the use of disposable dilation kits, balloon dilation kits, and/ or double-lumen tubes could resolve these issues and aid in achieving nephrostomy tract safety, they require additional costs. We subsequently focused on the structure of an inner ureteral access sheath (i-UAS). In ECIRS, we used either Bi-Flex $^{\text {TM }}$ UAS or Navigator ${ }^{\text {TM }}$ UAS during URS. As the former i-UAS has a double-lumen channel, we discovered the unique idea to utilize this i-UAS as not only a dilator but also a double-lumen catheter to insert a safety guidewire instead of using a dilation kit and/or double-lumen tube during the creation of the nephrostomy tract.

In the current study, we evaluated the safety and efficacy of using an i-UAS in ECIRS as a dilator and a double-lumen catheter and compared the outcomes with those of the conventional one-shot dilation technique. We hypothesized that the use of an i-UAS could be safer and more efficient than the conventional method. We present the following article in accordance with the STROBE reporting checklist (available at https://dx.doi.org/10.21037/tau-21-611).

\section{Methods}

\section{Study design}

This was a single-center non-randomized cohort study at the Nagoya City University Hospital, which was conducted from January 2016 to April 2020. Patients who underwent ECIRS due to large kidney and proximal ureteral stones were enrolled in this study. Most of the surgeries were performed by resident surgeons under the instructions of certified urologists. We compared the outcomes for percutaneous tract dilation performed using a conventional one-shot dilation (one-shot group) collected before July 2018 with those using i-UAS dilation (i-UAS group) collected after July 2018. We analyzed patients' demographics encompassing performance status (PS), sex, age, body mass index (BMI), and history of diabetes mellitus $(\mathrm{DM})$, and perioperative information, including details of preoperative antibiotic use, indwelling ureter stents, and nephrostomy, pyelonephritis, hydronephrosis (G0), main stone location [upper pole, middle pole, lower pole, and pelvis/ureteropelvic junction (UPJ)], staghorn stone, total stone number, stone density and volume (assessed by calculating length $\times$ width $\times$ depth $\times 0.167$ ), and surgery position.

Furthermore, we collected the operative and postoperative data regarding operative time (time from draping to wound dressing), fluoroscopy time, tract creation troubles (including tract loss and pelvic injuries during tract creation), urinary injury (defined as mucosal injury in the renal pelvis and calyx and perforations during PCNL), postoperative pyelonephritis, decrease in the hemoglobin level, duration of hospitalization, the indwelling of ureter stent and nephrostomy, additional surgical interventions as well as SFR after 3 months, which was defined as the absence of residual stones based on the computed tomography findings, and hereafter referred to as SFR (3M).

We evaluated the association between the use of i-UAS as a dilator and a double-lumen catheter and perioperative parameters. The primary endpoint was urinary injury and postoperative pyelonephritis, and the secondary endpoints were SFR (3M), operative time, fluoroscopy time, and duration of hospitalization. The study was conducted in accordance with the Declaration of Helsinki (as revised in 2013). The study was approved by the ethical committee of the Nagoya City University (60-19-0044, 60-19-0083) and informed consent was taken from all the patients.

\section{Surgical methods}

For ECIRS, we constructed a nephrostomy and performed surgery using the following methods: (I) patients were oriented in the prone split-leg position or modified Valdivia position (the position was chosen based on the surgeon's discretion considering the main stone location, staghorn 


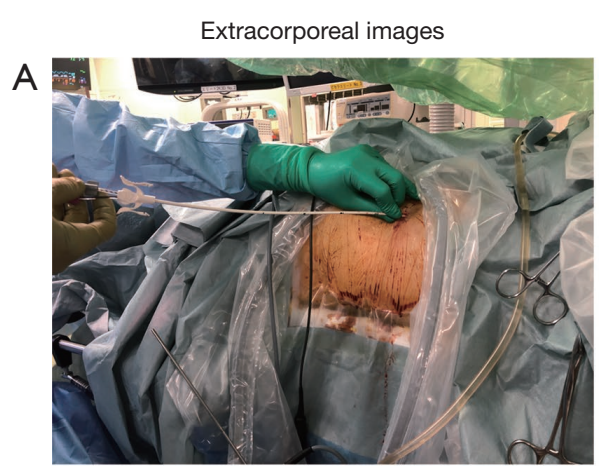

B

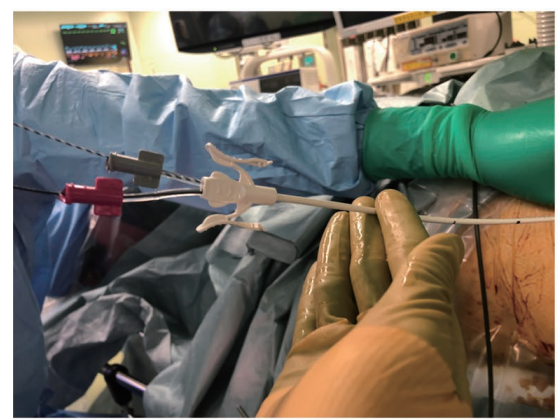

C

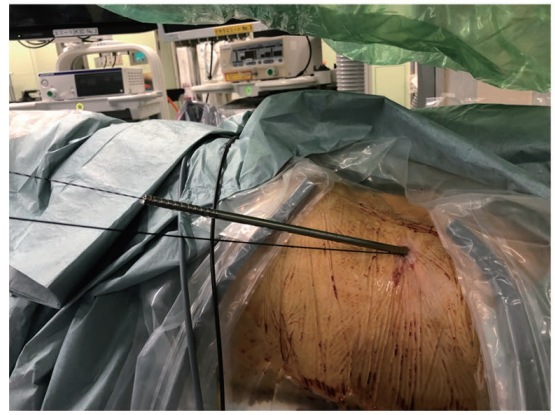

Endoscopic images
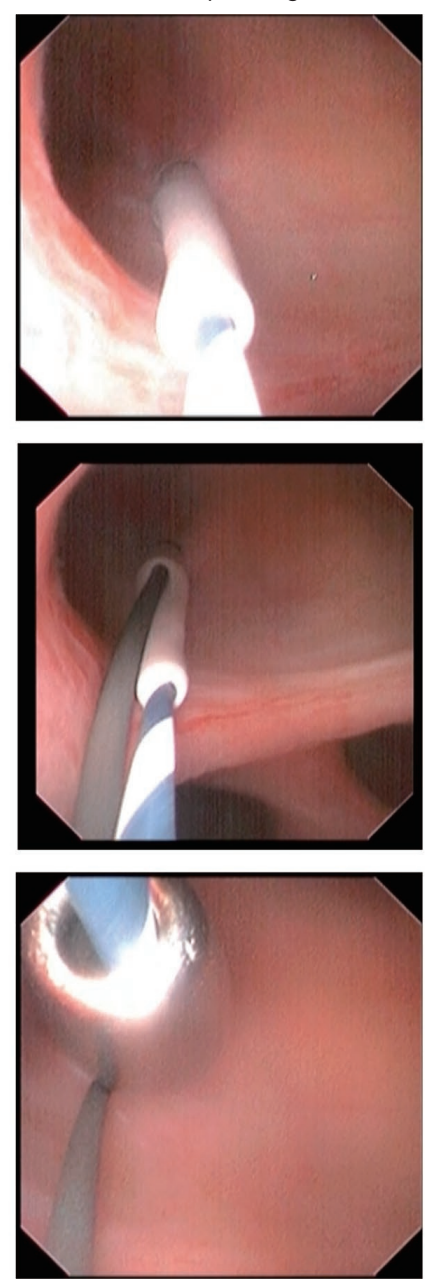

Fluoroscopic images
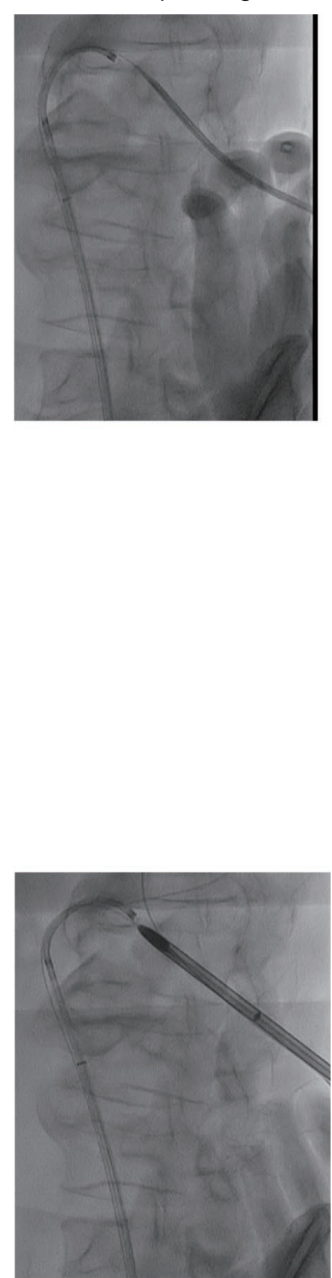

Figure 1 Dilation and insertion of a safety guidewire using an i-UAS. In the i-UAS group, we inserted i-UAS along the guidewire for dilation (A), and a safety guidewire was inserted through the double-lumen channel (B); further, we could insert a main tract along the guidewire (C). i-UAS, inner ureteral access sheath.

stone, and infection risk); (II) one urologist performed retrograde intrarenal surgery by using a flexible ureteroscope through a 10/12- or 12/14-Fr UAS (Bi-Flex ${ }^{\mathrm{TM}}$, Rocamed, Monaco; generally selecting $35 \mathrm{~cm}$ for females and $45 \mathrm{~cm}$ for males) in the i-UAS group and a $11 / 13$ - or 12/14-Fr UAS (Navigator ${ }^{\mathrm{TM}}$, Boston Scientific, MA, USA; generally selecting $36 \mathrm{~cm}$ for females and $46 \mathrm{~cm}$ for males) in the one-shot group, whereas another urologist performed renal puncture using a 22-gauge nephrostomy needle under US guidance; (III) the puncture of the nephrostomy needle into the collecting systems was monitored under direct ureteroscopic vision as much as possible, and we punctured repeatedly until we confirmed that the renal calyx had been punctured precisely; (IV) in the i-UAS group, we inserted the i-UAS with a double lumen along the guidewire for dilation, and a safety guidewire was inserted through another lumen; (V) a percutaneous tract $(16.5 / 17.5$ or 16.5/19.5 Fr; KARL STORZ, Tuttlingen, Germany) was inserted to establish working access (Figure 1); (VI) in the one-shot group, after successful puncturing, a single-step dilation using a $16.5 / 17.5$ - or a $16.5 / 19.5$-Fr metal tract was performed; (VII) after inserting the safety guidewire, we removed the percutaneous tract immediately and reinserted the percutaneous tract along the other (non-safety) guidewires (Figure 2); (VIII) after inserting the percutaneous tract, each urologist worked simultaneously to fragment and wash out the renal calculi using laser and Swiss LithoClast ${ }^{\circledR} 2$ (Boston Scientific) and a 12-Fr MIP-M nephroscope (KARL 

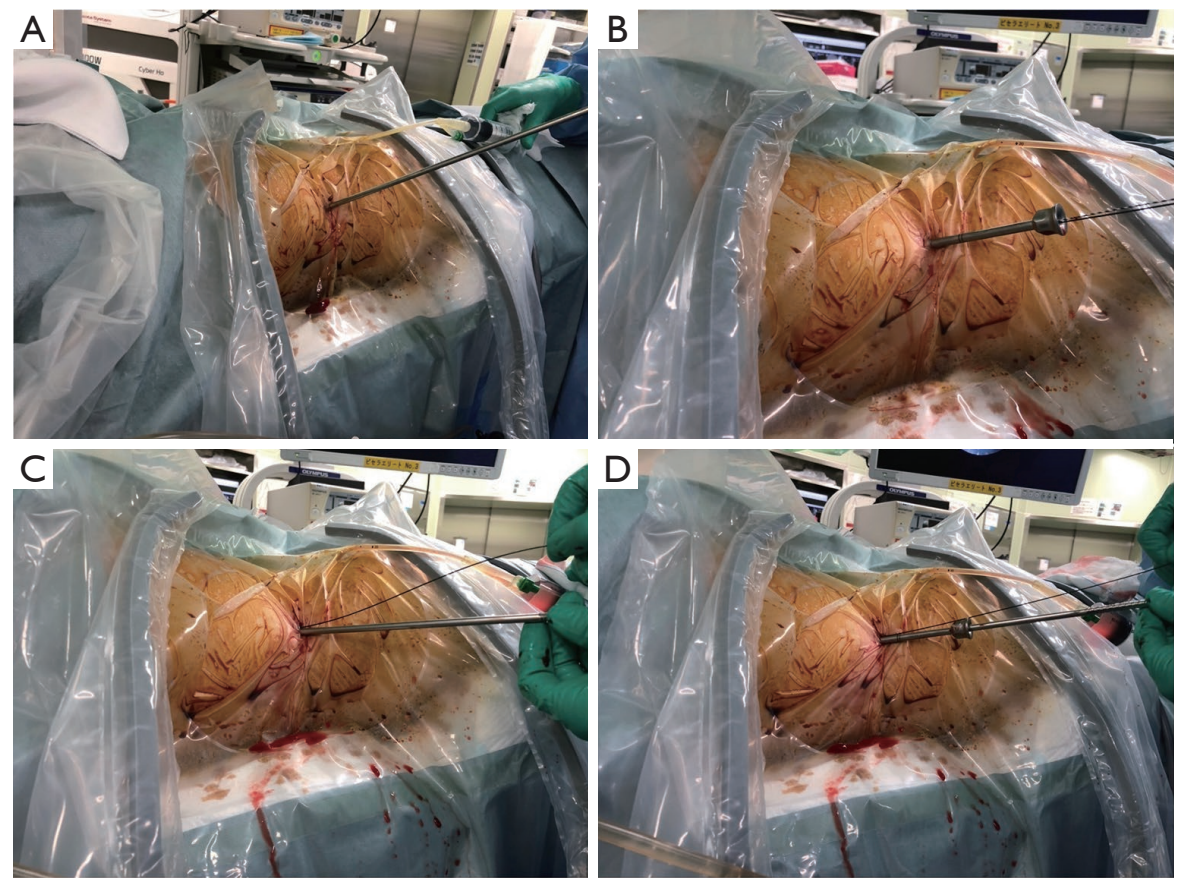

Figure 2 Insertion of a safety guidewire in the one-shot group. In the one-shot group, after performing a single-step dilation, we inserted a safety guidewire as well as the other guidewire thorough the tract $(\mathrm{A}, \mathrm{B})$. Thereafter, we removed the percutaneous tract, re-inserted it along the other (non-safety) guidewire (C), and inserted an outer tract along the inner tract (D).

STORZ); and (IX) at the end of surgery, a nephrostomy tube and/or ureteral catheter was inserted.

\section{Statistical analysis}

Continuous, normally distributed variables were expressed as mean \pm standard deviation, and non-normally distributed variables as median [25\% interquartile range (IQR), $75 \%$ IQR]. Categorical variables were presented as proportions. Student's $t$-test and the Mann-Whitney $\mathrm{U}$ test were used for continuous data, and chi-squared and Fisher's exact tests for categorical data. Multivariate logistic and linear regression analyses were performed and adjusted for age, BMI, sex, i-UAS use, preoperative non-hydronephrosis, stone density, stone volume, pelvic and UPJ stone, staghorn stone, total stone number $\geq 5$, spine position, and history of $\mathrm{DM}$, to identify the factors associated with perioperative urinary injury, postoperative pyelonephritis, SFR (3M), decrease in the postoperative hemoglobin level, operative time, fluoroscopy time, and duration of hospitalization. A $\mathrm{P}$ value $<0.05$ was considered statistically significant. All statistical analyses were performed using EZR (Saitama Medical Center, Jichi Medical University, Saitama, Japan), which is a graphical user interface for $\mathrm{R}$ (The R Foundation for Statistical Computing, Vienna, Austria) (16).

\section{Results}

A total of 221 patients were enrolled during the study period. Of these, 108 were in the i-UAS group, and 113 were in the one-shot group. Approximately $95 \%$ of the patients had a PS score of 0 . There were no significant differences between the two groups in patients' history of $\mathrm{DM}$, age, sex, BMI, preoperative antibiotics use, ureter stent, nephrostomy, pyelonephritis, hydronephrosis, stone location, staghorn stone, or total stone number. In the i-UAS group, the stone density was smaller and the modified Valdivia position was selected more often (Table 1).

In terms of operative and postoperative details, the operative time was shorter in the i-UAS group. No differences in tract creation troubles $(\mathrm{P}=0.05)$, postoperative pyelonephritis, decrease in the hemoglobin level, indwelling ureter stents, nephrostomy, duration of hospitalization, additional surgical intervention, and SFR (3M) were observed between the two groups (Table 2). There were no cases of failure when creating a nephrostomy tract with i-UAS. 
Table 1 Patient demographics and perioperative information

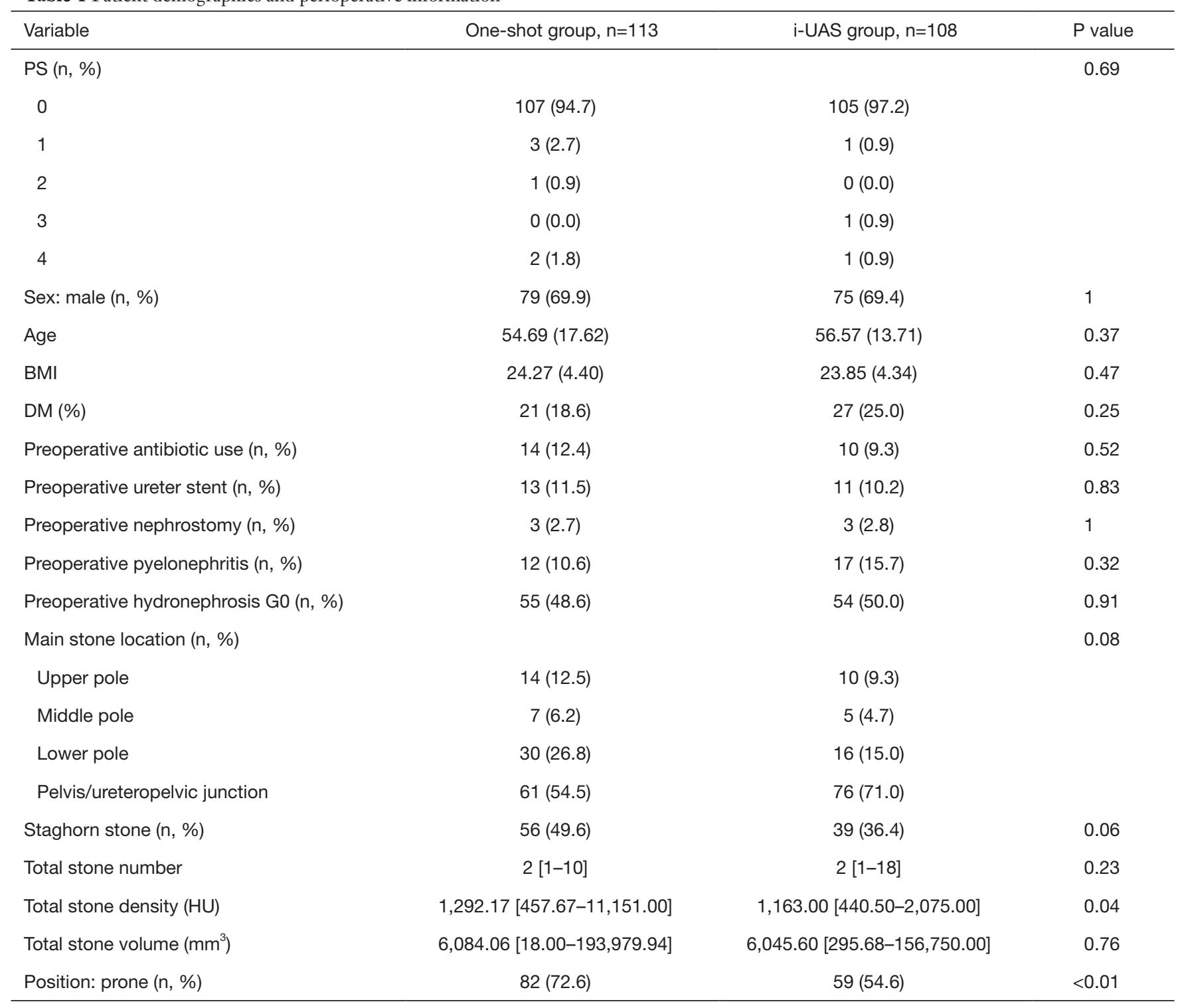

i-UAS, inner ureteral access sheath; PS, performance status; BMI, body mass index; DM, diabetes mellitus.

Multivariate analyses revealed that the use of i-UAS as a dilator and a double-lumen catheter to insert a safety guidewire was not significantly associated with complications (e.g., urinary injury, postoperative pyelonephritis), fluoroscopy time, and duration of hospitalization. However, the use of i-UAS was a strong factor for a shorter operative time and reduced tract creation troubles. Moreover, staghorn stone, total stone number $\geq 5$, higher stone density, and higher stone volume were significantly associated with a longer operative time (Tables 3,4). Our results indicated that older patients had a greater chance of operative injuries and male patients required longer hospitalization. Further, the modified Valdivia position contributed to lower chances of urinary injuries and postoperative pyelonephritis.

\section{Discussion}

PCNL is the standard treatment for renal stones larger than $20 \mathrm{~mm}$ (17-19); however, it contributes to increasing the risk of severe perioperative complications (7). To reduce complications with PCNL, minimally invasive PCNL (mini- and ultra-mini PCNL) (8-10) and ECIRS 
Table 2 Operative and postoperative information

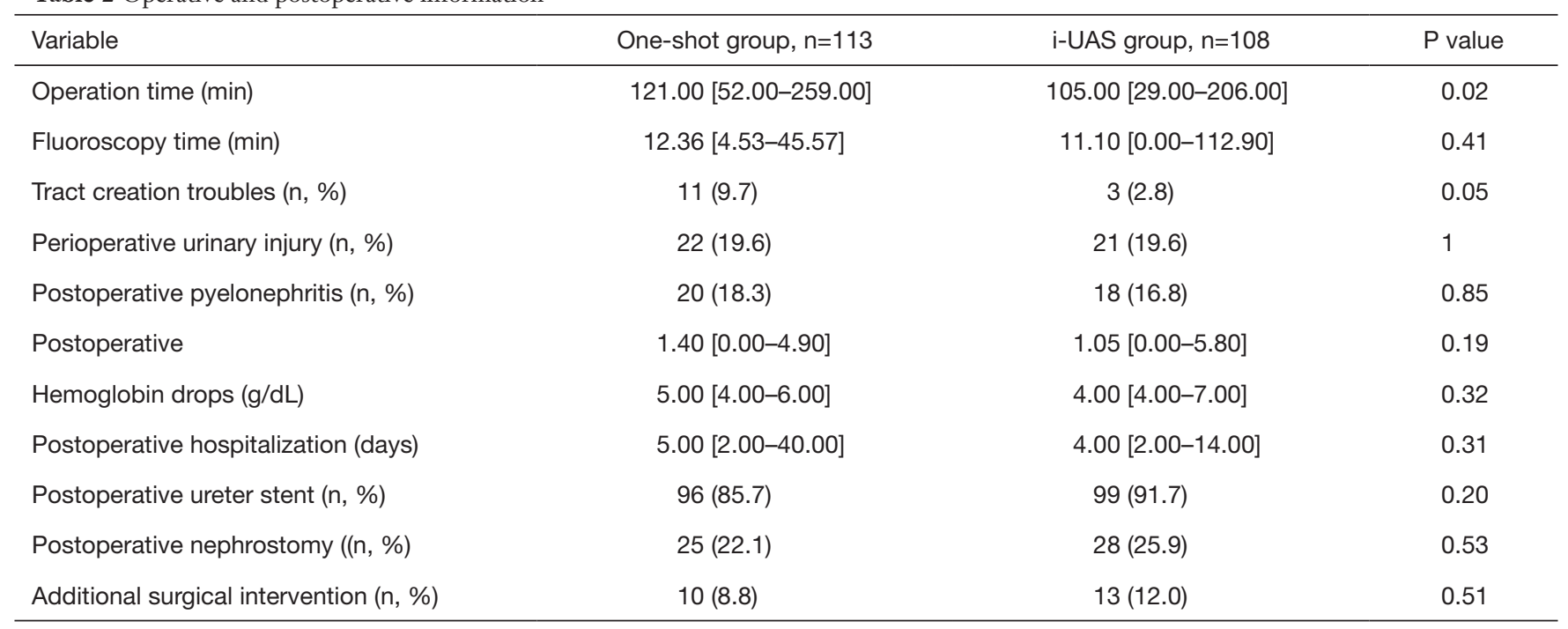

i-UAS, inner ureteral access sheath.

Table 3 Multivariate analyses of logistic regression models (tract creation trouble, perioperative urinary injury, postoperative pyelonephritis

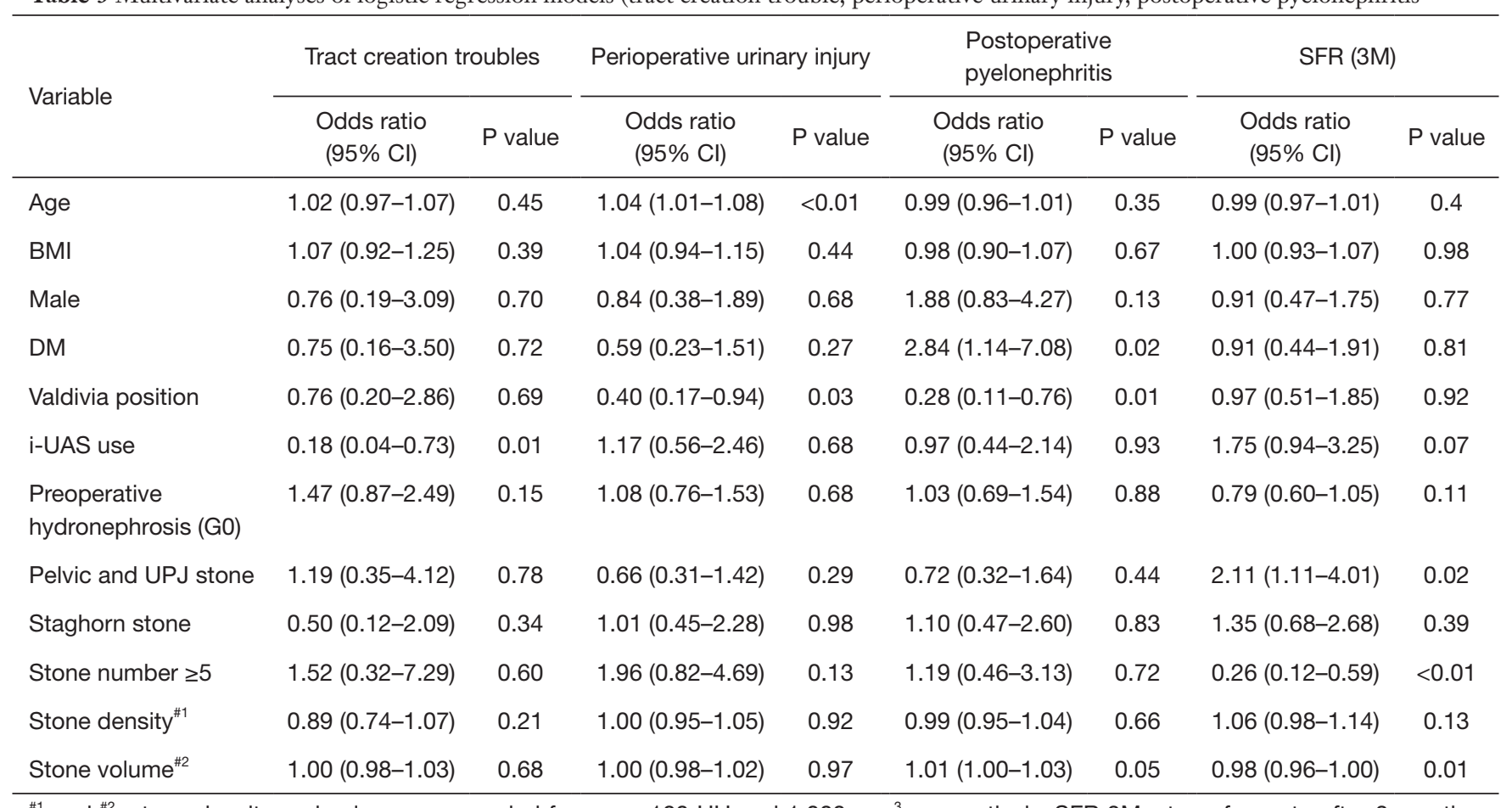

${ }^{\# 1}$ and ${ }^{\# 2}$ : stone density and volume were scaled for every $100 \mathrm{HU}$ and 1,000 $\mathrm{mm}^{3}$, respectively. SFR 3M, stone-free rate after 3 months;

$\mathrm{Cl}$, confidence interval; DM, diabetes mellitus; i-UAS, inner ureteral access sheath; UPJ, ureteropelvic junction.

with the simultaneous combined use of fURS and PCNL were developed (2). ECIRS has been reported to cause less bleeding than PCNL alone (20-22); nonetheless, the corresponding incidence of complications ranges from
$10.0 \%$ to $48.4 \%$ (3-6). Obtaining safe percutaneous renal access is, therefore, an important step in PCNL and ECIRS to reduce complications.

In this study, to further reduce complications, we 


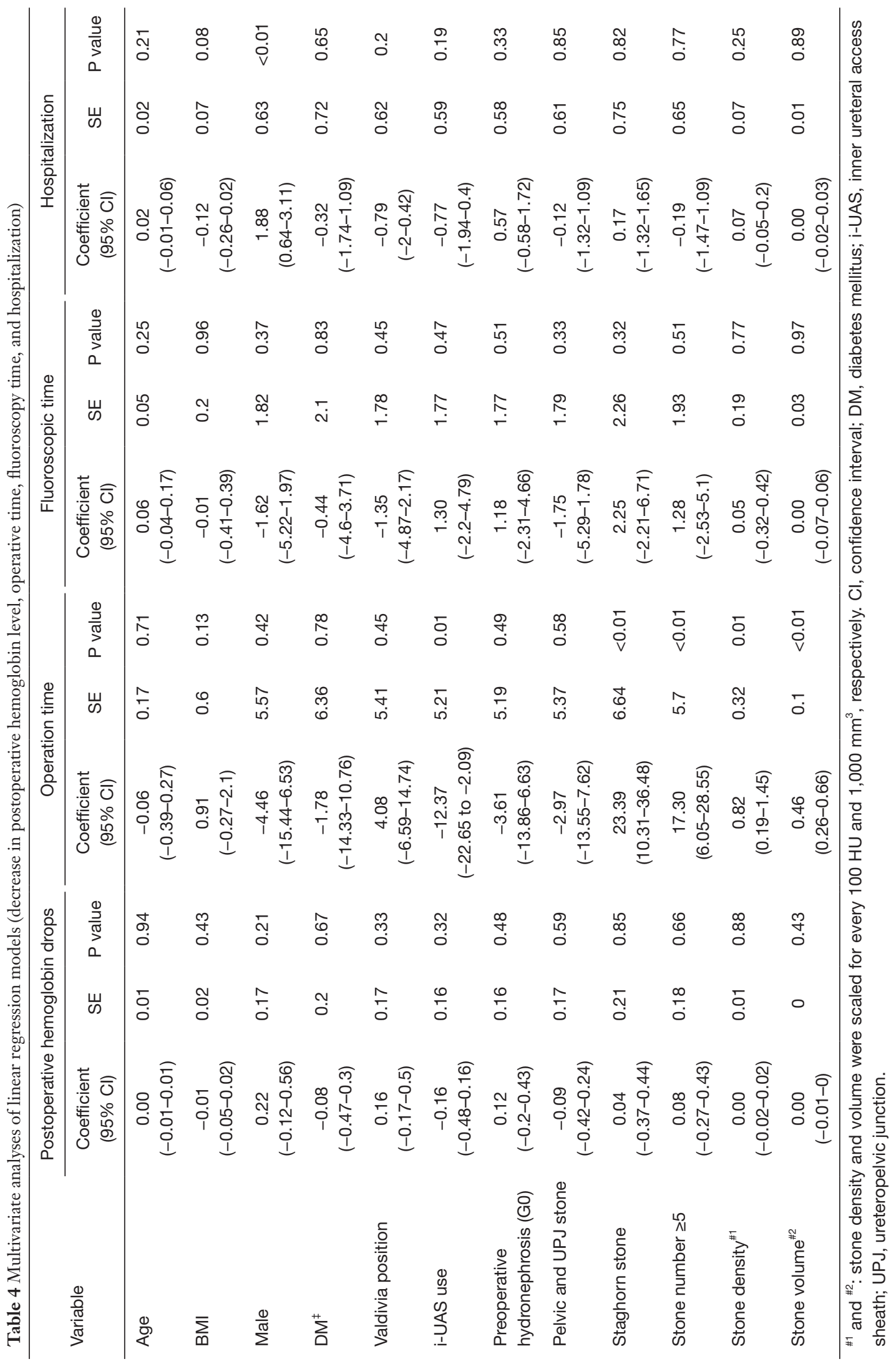


examined the safety and efficacy of using an i-UAS with double-lumen channels for the dilation and insertion of a safety guidewire when creating a nephrostomy tract. This technique reduced tract creation troubles and did not cause any further perioperative complications compared with previous methods. Consistent with current studies, a history of DM was identified as a predictor for an increased risk of postoperative infection. We also found staghorn stone, a larger number of stones, higher stone density, and higher stone volume as strong factors associated with longer operative time, while the use of i-UAS significantly decreased the operative time. The use of i-UAS showed no association with the increase in fluoroscopy time and duration of hospitalization as well as with the decrease in SFR (3M).

Because most ECIRS procedures at our institute were performed by young doctors under the instructions of certified urologists, a safe protocol for creating the nephrostomy tract was required. Compared to the conventional dilation method using the dilation kit or balloon dilator, one-shot dilation is more likely to decrease the fluoroscopy time and bleeding amount and is currently becoming a common technique in creating percutaneous tracts. However, this technique might be associated with renal parenchymal damage, microvasculature damage, and a lower successful dilation rate due to a lack of surgical experience (13-15). Given that approximately 60 cases are needed as a learning curve for competence in percutaneous renal access $(23,24)$, the selection of one-shot dilation by less-experienced surgeons remains debatable. Therefore, to reduce nephrostomy tract-related morbidity, the use of dilator kits might be an option for inexperienced doctors and in cases with high resistance due to perinephric scar tissue (25). Although utilizing dilator kits was helpful for the procedure, this requires additional cost. The use of certain types of dilators, such as the Amplatz-type renal dilator and NephroMax ${ }^{\mathrm{TM}}$ High Pressure Nephrostomy Balloon Catheter Kit, requires an extra cost of almost 400 US dollars per application. Further, for alternative methods, we used i-UAS with a double-lumen sheath as a dilator. In over $90 \%$ of ECIRS cases, we selected a tract (outer sheath) of $17.5 \mathrm{Fr}$ or greater. Therefore, we considered that tract placement could be performed more safely using $10-\mathrm{Fr}$ or 12-Fr i-UAS as a dilator without extra cost. The results showed that dilation with an i-UAS was not associated with urinary injury, decrease in the postoperative hemoglobin level, and longer fluoroscopy time, but was associated with a lower chance of tract creation troubles.
Additionally, the placement of a safety guidewire could be an option for safely performing procedures. The American Urological Association guidelines recommend the use of a safety guidewire when performing PCNL, in case the primary wire is lost or displaced or the collecting system is injured (18). Recent studies have described that only $56 \%$ of surgeons in high-case-volume hospitals with $>100$ cases of PCNL per year use a safety guidewire, compared to the $84 \%$ of urologists in low-case-volume hospitals having $<50$ cases who utilize it (26), indicating that inexperienced surgeons are more likely to place the safety guidewire. Given these facts, we adopted the technique of placing safety guidewires during the creation of the nephrostomy tract. Despite its role in safety, the placement of a safety guidewire during one-shot dilation carries an increased risk of urinary injury and bleeding owing to the removal of the tract and re-insertion after the placement of the safety guidewire.

Using a double-lumen tube might help resolve this issue; however, it entails the additional cost of disposable tubes or hospital resources in terms of sterilization of reusable ones. For example, the cost of a dual lumbar ureteral access catheter (Cook Medical, IN, USA) is 80 US dollars per application. Based on our results, using an i-UAS with double-lumen channels enabled us to simultaneously place safety guidewires without extra cost and did not contribute to an increase in perioperative complications. For reference, the prices of other UASs are 410 US dollars for Bi-Flex ${ }^{\text {TM, }}$ 390 US dollars for Navigator ${ }^{\mathrm{TM}}$, and 530 US dollars for Flexor ${ }^{\mathrm{TM}}$ Ureteral Access Sheath (Cook Medical). When we use both a commercial base dilator and a dual-lumen catheter, the maximal total cost is found to be 870 US dollars (when using a Navigator ${ }^{\mathrm{TM}}$ ) and 1010 US dollars (when using a Flexor ${ }^{\mathrm{TM}}$ ), respectively (Table 5). Considering the absence of substantial price differences, selecting i-UAS with a double-lumen channel for the URS side has a safe and efficient role in creating nephrostomy for the PCNL side.

Furthermore, the current study also showed that the use of i-UAS was associated with a decrease in the operative time. This is uncertain due to the lack of exact time recorded for the tract creation, and the simultaneous placement of the safety guidewire without a replacement tract could contribute to a shorter operative time. On the contrary, the conventional method of one-step dilation requires an additional insertion of the inner metal tract after replacing a safety guidewire through the outer metal sheath. This double insertion of the inner metal 
Table 5 Cost comparison between the one-shot group and the i-UAS group

\begin{tabular}{|c|c|c|c|}
\hline Variable & \multicolumn{2}{|c|}{ One-shot group } & $\frac{\mathrm{i}-\mathrm{UAS} \text { group }}{\mathrm{Bi}^{\mathrm{Flex}}{ }^{\mathrm{TM}}}$ \\
\hline Access sheath, price (US dollars) & 390 & 530 & 410 \\
\hline \multicolumn{4}{|l|}{ If using dual lumbar catheter and/or dilator kit } \\
\hline Dual lumbar catheter, price (US dollars) & 80 & 80 & Use i-UAS \\
\hline NephroMax ${ }^{\mathrm{TM}}$, price (US dollars) & 400 & 400 & Use i-UAS \\
\hline Maximum total cost* & 870 & 1,010 & 410 \\
\hline
\end{tabular}

*, access sheath + dual lumbar catheter + Amplatz dilator or NephroMax. i-UAS, inner ureteral access sheath.

tract is difficult and could cause complications, including bleeding or misplacement of the inner tract, for young doctors with little experience and can be time-consuming. This is beneficial because prolonged operative time has been reported to increase the risk of infection (27). The occurrence of lower SFR, longer operative time, and longer hospitalization periods are mainly dependent on stone density and volume.

Our study has some limitations. First, when designing this study, we did not perform randomization when grouping the patients. Furthermore, this study was a singlecenter cohort study, which may have resulted in biases, such as facility-specific surgery cases. Second, although most surgeries were mainly performed by residents, there was a potential bias as some of the differences in outcomes may be considered due to a learning curve, considering that the operations in the two groups were performed during different periods. Comparing the procedures between 2017-2018 in the one-shot group $(n=50)$ and 2018-2019 in the i-UAS group $(n=50)$, there were no significant differences in terms of patients' demographics. However, the operative time was still shorter in the i-UAS group (Table S1), suggesting that the learning curve might not affect the results. Moreover, because the surgical procedure may differ depending on the timing of surgery, in the i-UAS group, the stone density was smaller, and the modified Valdivia position was selected more frequently; this may have significantly influenced the outcomes. Finally, as we did not examine the presence of fluoroscopy time, and procedure time during tract creation, we could not compare the direct effects of using an i-UAS on tract creation.

In conclusion, we found that the use of an i-UAS as a dilator and a double-lumen catheter to insert a safety guidewire during ECIRS is a convenient and safe technical method that can reduce the operative time. These results, which are from real-world data and reflect our daily practice, require further validation. However, they have technical ingenuity for creating a nephrostomy tract, especially for surgeries with a relatively short operative time.

\section{Acknowledgments}

Funding: None.

\section{Footnote}

Reporting Checklist: The authors have completed the STROBE reporting checklist. Available at https://dx.doi. org/10.21037/tau-21-611

Data Sharing Statement: Available at https://dx.doi. org/10.21037/tau-21-611

Peer Review File: Available at https://dx.doi.org/10.21037/ tau-21-611

Conflicts of Interest: All authors have completed the ICMJE uniform disclosure form (available at https://dx.doi. org/10.21037/tau-21-611). The authors have no conflicts of interest to declare.

Ethical Statement: The authors are accountable for all aspects of the work in ensuring that questions related to the accuracy or integrity of any part of the work are appropriately investigated and resolved. The study was conducted in accordance with the Declaration of Helsinki (as revised in 2013). The study was approved by the ethical committee of the Nagoya City University (60-19-0044, 
60-19-0083) and informed consent was taken from all the patients.

Open Access Statement: This is an Open Access article distributed in accordance with the Creative Commons Attribution-NonCommercial-NoDerivs 4.0 International License (CC BY-NC-ND 4.0), which permits the noncommercial replication and distribution of the article with the strict proviso that no changes or edits are made and the original work is properly cited (including links to both the formal publication through the relevant DOI and the license). See: https://creativecommons.org/licenses/by-nc-nd/4.0/.

\section{References}

1. Ruhayel Y, Tepeler A, Dabestani S, et al. Tract Sizes in Miniaturized Percutaneous Nephrolithotomy: A Systematic Review from the European Association of Urology Urolithiasis Guidelines Panel. Eur Urol 2017;72:220-35.

2. Scoffone CM, Cracco CM, Cossu M, et al. Endoscopic combined intrarenal surgery in Galdakao-modified supine Valdivia position: a new standard for percutaneous nephrolithotomy? Eur Urol 2008;54:1393-403.

3. Kawahara T, Ito H, Terao H, et al. Ureteroscopy assisted retrograde nephrostomy: a new technique for percutaneous nephrolithotomy (PCNL). BJU Int 2012;110:588-90.

4. Marguet CG, Springhart WP, Tan YH, et al. Simultaneous combined use of flexible ureteroscopy and percutaneous nephrolithotomy to reduce the number of access tracts in the management of complex renal calculi. BJU Int 2005;96:1097-100.

5. Wen J, Xu G, Du C, et al. Minimally invasive percutaneous nephrolithotomy versus endoscopic combined intrarenal surgery with flexible ureteroscope for partial staghorn calculi: A randomised controlled trial. Int J Surg 2016;28:22-7.

6. Zhong W, Zhao Z, Wang L, et al. Percutaneous-based management of Staghorn calculi in solitary kidney: combined mini percutaneous nephrolithotomy versus retrograde intrarenal surgery. Urol Int 2015;94:70-3.

7. Suarez-Ibarrola R, Desai JD. Is mini-percutaneous nephrolithotomy the way to go for renal stones? Yes! Curr Opin Urol 2019;29:309-11.

8. Jackman SV, Hedican SP, Peters CA, et al. Percutaneous nephrolithotomy in infants and preschool age children: experience with a new technique. Urology 1998;52:697-701.

9. Wright A, Rukin N, Smith D, et al. 'Mini, ultra, micro'
- nomenclature and cost of these new minimally invasive percutaneous nephrolithotomy (PCNL) techniques. Ther Adv Urol 2016;8:142-6.

10. Desai J, Zeng G, Zhao Z, et al. A novel technique of ultramini-percutaneous nephrolithotomy: introduction and an initial experience for treatment of upper urinary calculi less than $2 \mathrm{~cm}$. Biomed Res Int 2013;2013:490793.

11. Sugino T, Hamamoto S, Unno R, et al. Effectiveness of ureteroscope-assisted renal puncture for endoscopic combined intrarenal surgery. Int J Urol 2019;26:424-5.

12. Hamamoto S, Unno R, Taguchi K, et al. A New Navigation System of Renal Puncture for Endoscopic Combined Intrarenal Surgery: Real-time Virtual Sonography-guided Renal Access. Urology 2017;109:44-50.

13. Srivastava A, Singh S, Dhayal IR, et al. A prospective randomized study comparing the four tract dilation methods of percutaneous nephrolithotomy. World J Urol 2017;35:803-7.

14. Aminsharifi A, Alavi M, Sadeghi G, et al. Renal parenchymal damage after percutaneous nephrolithotomy with one-stage tract dilation technique: a randomized clinical trial. J Endourol 2011;25:927-31.

15. Wu Y, Xun Y, Lu Y, et al. Effectiveness and safety of four tract dilation methods of percutaneous nephrolithotomy: A meta-analysis. Exp Ther Med 2020;19:2661-71.

16. Kanda Y. Investigation of the freely available easy-touse software 'EZR' for medical statistics. Bone Marrow Transplant 2013;48:452-8.

17. European Association of Urology. European Association of Urology Guidelines. 2019 Edition. Vol presented. European Association of Urology Guidelines Office, 2020.

18. Assimos D, Krambeck A, Miller NL, et al. Surgical Management of Stones: American Urological Association/ Endourological Society Guideline, PART I. J Urol 2016;196:1153-60.

19. Taguchi K, Cho SY, Ng AC, et al. The Urological Association of Asia clinical guideline for urinary stone disease. Int J Urol 2019;26:688-709.

20. Kuroda S, Ito H, Sakamaki K, et al. Development and Internal Validation of a Classification System for Predicting Success Rates After Endoscopic Combined Intrarenal Surgery in the Modified Valdivia Position for Large Renal Stones. Urology 2015;86:697-702.

21. Scoffone CM, Cracco CM, Poggio M, et al. Endoscopic combined intrarenal surgery for high burden renal stones. Arch Ital Urol Androl 2010;82:41-2.

22. Hamamoto S, Yasui T, Okada A, et al. Developments in the technique of endoscopic combined intrarenal surgery 
in the prone split-leg position. Urology 2014;84:565-70.

23. Quirke K, Aydin A, Brunckhorst O, et al. Learning Curves in Urolithiasis Surgery: A Systematic Review. J Endourol 2018;32:1008-20.

24. legislation.gov.uk [internet]. The Working Time Regulations. SI 1988 No. 1833. [cited]. Available online: http://www.legislation.gov.uk/uksi/1998/1833/ contents/made

25. Falahatkar S, Neiroomand H, Akbarpour M, et al. One-

Cite this article as: Unno R, Taguchi K, Hamamoto S, Hattori T, Kawase K, Okada T, Chaya R, Tanaka Y, Sugino T, Kato T, Etani T, Ando R, Okada A, Yasui T. A novel approach in creating nephrostomy using a double-lumen access sheath during endoscopic combined intrarenal surgery. Transl Androl Urol 2021;10(11):4181-4191. doi: 10.21037/tau-21-611 shot versus metal telescopic dilation technique for tract creation in percutaneous nephrolithotomy: comparison of safety and efficacy. J Endourol 2009;23:615-8.

26. Ahmad AA, Alhunaidi O, Aziz M, et al. Current trends in percutaneous nephrolithotomy: an internet-based survey. Ther Adv Urol 2017;9:219-26.

27. Cheng H, Chen BP, Soleas IM, et al. Prolonged Operative Duration Increases Risk of Surgical Site Infections: A Systematic Review. Surg Infect (Larchmt) 2017;18:722-35. 


\section{Supplementary}

Table S1 Patient demographics and perioperative data (operative time and tract creation trouble) between 2017-2018 in the one-shot group ( $\mathrm{n}=50$ ) and 2018-2019 in the i-UAS group $(\mathrm{n}=50)$

\begin{tabular}{|c|c|c|c|}
\hline Variable & One-shot group [2017-2018], n=50 & i-UAS group [2018-2019], $\mathrm{n}=50$ & $P$ value \\
\hline 0 & $47(94.0)$ & $48(96.0)$ & 1 \\
\hline 1 & $2(4.0)$ & $1(2.0)$ & \\
\hline 2 & $0(0.0)$ & $0(0.0)$ & \\
\hline 4 & $1(2.0)$ & $1(2.0)$ & \\
\hline Sex: male (\%) & $16(32.0)$ & $21(42.0)$ & 0.40 \\
\hline Age & $55.80(18.45)$ & $56.12(14.85)$ & 0.92 \\
\hline BMI & $24.67(4.71)$ & $23.34(5.12)$ & 0.18 \\
\hline Preoperative ureter stent (\%) & $5(10.0)$ & $5(10.0)$ & 1 \\
\hline Preoperative nephrostomy (\%) & $2(4.0)$ & $1(2.0)$ & 1 \\
\hline Preoperative pyelonephritis (\%) & $4(8.0)$ & $7(14.0)$ & 0.52 \\
\hline Preoperative Hydronephrosis G0 (\%) & $5(10.0)$ & $5(10.0)$ & 1 \\
\hline \multicolumn{4}{|l|}{ Main stone location (\%) } \\
\hline Upper pole & $10(20.4)$ & $4(8.0)$ & 0.24 \\
\hline Middle pole & $2(4.1)$ & $3(6.0)$ & \\
\hline Lower pole & $10(20.4)$ & $8(16.0)$ & \\
\hline Position: prone (\%) & $36(72.0)$ & $33(66.0)$ & 0.66 \\
\hline Operation time (min) & 119.00 [61.00-259.00] & 104.00 [48.00-186.00] & 0.04 \\
\hline Tract creation trouble (\%) & $4(8.0)$ & $2(4.0)$ & 0.67 \\
\hline
\end{tabular}

i-UAS, inner ureteral access sheath; PS, performance status; BMI, body mass index; DM, diabetes mellitus. 